\title{
Correction: Unibody Endograft Using AFX 2 for Less Invasive and Faster Endovascular Aortic Repair: Protocol for a Multicenter Nonrandomized Study
}

Roberto Silingardi $^{1 *}$, MD; Pasqualino Sirignano ${ }^{2 *}$, MD; Francesco Andreoli ${ }^{1}$, MD; Wassim Mansour ${ }^{2}, \mathrm{MD}, \mathrm{PhD}$; Mattia Migliari $^{1}$, MD; Francesco Speziale ${ }^{2}$, MD; LIVE Study Collaborators ${ }^{3 *}$

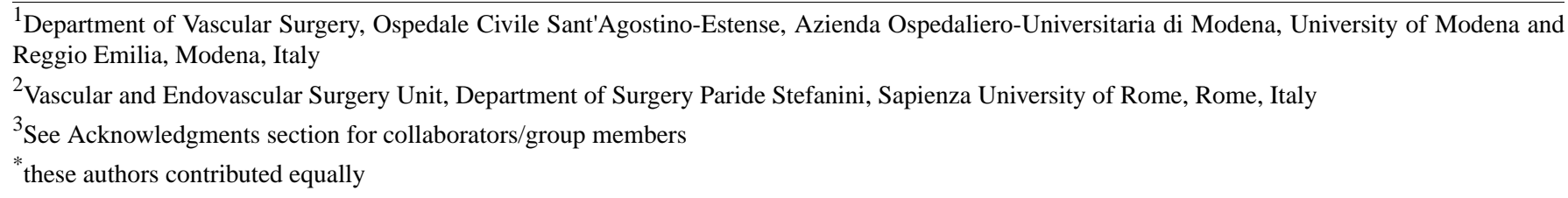

\section{Corresponding Author:}

Pasqualino Sirignano, MD

Vascular and Endovascular Surgery Unit

Department of Surgery Paride Stefanini

Sapienza University of Rome

Viale del Policlinico, 155

Rome, 00161

Italy

Phone: 39064940532

Email: pasqualino.sirignano@uniroma1.it

\section{Related Article:}

Correction of: https://www.researchprotocols.org/2020/4/e16959/

(JMIR Res Protoc 2020;9(6):e20698) doi: 10.2196/20698

In "Unibody Endograft Using AFX 2 for Less Invasive and Faster Endovascular Aortic Repair: Protocol for a Multicenter Nonrandomized Study" (JMIR Res Protoc 2020;9(4):e16959) there were two errors in the Collaborators List.

The collaborator Sonia Ronchey was inadvertently not included in the collaborator list. Additionally, the collaborator name Pietro Volpet should have been listed as Pietro Volpe.

The Collaborator List was initially published as follows:

The LIVE Study Collaborators are as follows: Giancarlo Accarino; Dimitri Apostolou; Guido Bajardi; Stefano Bartoli; Filippo Benedetto; Franco Briolini; Stefano Camparini; Emidio Costantini; Giovanni Credi; Ruggiero Curci; Raffaello Dallatana; Gianmarco de Donato; Carlo Dionisi; Vittorio Dorrucci; Leonardo Ercolini; Gianfranco Fadda; Mauro Ferrari; Loris Flora; Andrea Gaggiano; Roberto Gattuso; Franco Grego; Sabrina Grimaldi; Giovanni Impedovo; Arnaldo Ippoliti; Antonio Jannello; Sergio Losa; Nicola Mangialardi; Isaac Martinez; Javier Martinez; Stefano Michelagnoli; Giancarlo Palasciano; Vincenzo Palazzo; Domenico Palombo; Raffaele Pulli; Giovanni Rossi; Antonino Scolaro; Gianantonio Simoni; Francesco Spinelli; Francesco Talarico; Maurizio Taurino; Marco Trogolo; Nicola Tusini;
Gianfranco Veraldi; Pier Francesco Veroux; Gennaro

Vigliotti; and Pietro Volpet.

The Collaborator List has now been updated to the following:

The LIVE Study Collaborators are as follows: Giancarlo Accarino; Dimitri Apostolou; Guido Bajardi; Stefano Bartoli; Filippo Benedetto; Franco Briolini; Stefano Camparini; Emidio Costantini; Giovanni Credi; Ruggiero Curci; Raffaello Dallatana; Gianmarco de Donato; Carlo Dionisi; Vittorio Dorrucci; Leonardo Ercolini; Gianfranco Fadda; Mauro Ferrari; Loris Flora; Andrea Gaggiano; Roberto Gattuso; Franco Grego; Sabrina Grimaldi; Giovanni Impedovo; Arnaldo Ippoliti; Antonio Jannello; Sergio Losa; Nicola Mangialardi; Isaac Martinez; Javier Martinez; Stefano Michelagnoli; Giancarlo Palasciano; Vincenzo Palazzo; Domenico Palombo; Raffaele Pulli; Sonia Ronchey; Giovanni Rossi; Antonino Scolaro; Gianantonio Simoni; Francesco Spinelli; Francesco Talarico; Maurizio Taurino; Marco Trogolo; Nicola Tusini; Gianfranco Veraldi; Pier Francesco Veroux; Gennaro Vigliotti; and Pietro Volpe.

The correction will appear in the online version of the paper on the JMIR website on June 24, 2020, together with the publication of this correction notice. Because this was made 
after submission to PubMed, PubMed Central, and other full-text those repositories. repositories, the corrected article has also been resubmitted to

This is a non-peer-reviewed article. Submitted 26.05.20; accepted 29.05.20; published 24.06.20.

Please cite as:

Silingardi R, Sirignano P, Andreoli F, Mansour W, Migliari M, Speziale F, LIVE Study Collaborators

Correction: Unibody Endograft Using AFX 2 for Less Invasive and Faster Endovascular Aortic Repair: Protocol for a Multicenter

Nonrandomized Study

JMIR Res Protoc 2020;9(6):e20698

URL: http://www.researchprotocols.org/2020/6/e20698/

doi: $\underline{10.2196 / 20698}$

PMID: $\underline{32579539}$

CRoberto Silingardi, Pasqualino Sirignano, Francesco Andreoli, Wassim Mansour, Mattia Migliari, Francesco Speziale, LIVE Study Collaborators. Originally published in JMIR Research Protocols (http://www.researchprotocols.org), 24.06.2020. This is an open-access article distributed under the terms of the Creative Commons Attribution License (https://creativecommons.org/licenses/by/4.0/), which permits unrestricted use, distribution, and reproduction in any medium, provided the original work, first published in JMIR Research Protocols, is properly cited. The complete bibliographic information, a link to the original publication on http://www.researchprotocols.org, as well as this copyright and license information must be included. 